Original Article

\title{
The Development of a Mental Toughness Scale for Doctors:
}

\section{A Psychometric Study}

\author{
Saba Aslam, Sadia Saleem, Zahid Mahmood
}

\begin{abstract}
OBJECTIVE: To develop a scale of mental toughness among medical doctors.

METHODOLOGY: In the current study Mixed-Method research design was used, qualitative design was used to elicit the items for mental toughness and quantitative was used to establish psychometrics. This study was carried out in Govt. hospitals of Lahore. The study was carried out from August 2018-April 2019. The construct of mental toughness was explored from 20 post graduate residents and house officers (10 men and 10 women) through a semi structured interview. After excluding the repetition finalist of 40 items was further validated by 10 experts and based on the $50 \%$ agreement from the experts, a list of 30 items was finalized and piloted on 40 doctors (20 men and 20 women) for assessing user friendliness. Finally, a sample of two hundred doctors (100 men and 100 Women) between the ages of 22 to 36 ( $M=26.17, S D=1.83$ ) from different Government. Hospitals of Lahore were given Mental Toughness Scale, Depression, Anxiety, Stress Scale (DASS-21) along with a Demographic form.

RESULTS: Exploratory factor analysis through varimax rotation yielded three factors solution named as Determination, Resilience and Optimism. The MTS was found to have sound psychometric properties as split half reliability was .74 test, retest reliability was .75 .

CONCLUSION: Mental toughness scale was found high psychometric properties; results were discussed in term of counseling and enhancing positive strengths in medical doctors.
\end{abstract}

KEY WORDS: Mental toughness, Culture, Medical doctors, Scale, Mental health.

This article may be cited as: Aslam S, Saleem S, Mahmood Z. The Development of a Mental Toughness Scale for Doctors: A Psychometric Study. J Liaquat Uni Med Health Sci. 2019;18(03): 214-8. doi: 10.22442/jlumhs.191830630

\section{INTRODUCTION}

The new trends and advancement in positive psychology are now focused on strength based model that enable person to strive ${ }^{1,2}$. Mental toughness is a one such personality construct, that how people deal with the stressors, pressures and challenges of life, irrespective of prevailing circumstances ${ }^{3}$ and interchangeably described with the constructs of resilience and hardiness ${ }^{4}$. However, resilience is a positive construct of coping and bouncing back from stressful situations ${ }^{5}$ while mental toughness is a personality construct, a person's ability to deal with the stress and challenges in daily routine life ${ }^{6}$. Mentally tough are said to be more consistent and persistent ${ }^{7,2}$, focused and passionate about their work ${ }^{8}$. Mental toughness is mostly studied with reference to sport performance of athletes ${ }^{9}$, yet few systematic attempts have been made to study mental toughness outside sports context ${ }^{10}$.

Since medical profession is considered as one of the most stressful profession where a great deal of public communication is involved ${ }^{11}$. Stressful working conditions, long working hours and dealing with emergencies make this profession as more stressful and may lead to serious mental health problems ${ }^{12,13}$.
Moreover, if these mental health problems are not identified early and intervene timely may lead to serious psychosocial consequences including burnout, depression, and unemployment ${ }^{14}$. Research evidence also suggest that not all medical professional seek help for mental health problems because of stigma associated with and remain suffering from mental health issues ${ }^{15,16}$.

Yet not all individuals experience the adverse consequences of job stress because of some of the protective factors such as mental toughness ${ }^{17}$. Mental toughness is related with quality of life of the people and healthy sleep pattern and duration ${ }^{18}$. The concept of mental toughness had attained very little attention in Pakistan especially with reference to medical profession ${ }^{19}$. Unfortunately no attempt was made to develop any measurement tool for mental toughness in doctors. This research would be pioneer in nature would help to understand the phenomena of mental toughness in cultural context and also to see the expression of mental toughness in doctors ${ }^{11}$.

\section{METHODOLOGY}

The current study Mixed-Method research qualitative design was used to elicit the items for mental 
toughness and quantitative was used to establish psychometrics.

This study was carried out in Government Hospitals of Lahore. The study was carried out from August 2018April 2019. A semi-structured interview technique was used to generate item pool using emic approach. Mental toughness was operationally defined as an individual's capacity that enables him to call upon inner abilities, skills and strengths to deal with demands of an aversive situation ${ }^{2}$. Operational definition of the construct translated into Urdu was given to the doctors and they were asked to provide the characteristic features of mental toughness in doctors. An initial list of 50 items was collected by asking question from postgraduate doctors and house officers (10 men and 10 women). As the interview progressed, repetition increased and only new statements retained. All interviews were recorded in verbatim and content analysis was carried out to generate item pooland list of 40 items was extracted by excluding ambiguity and repetition.

Operational definition was given to the ten experts and asked to rate each item to keep in mind the construct of mental toughness. The experts comprised 5 clinical psychologists and 5 medical doctors with 5 years of minimum experience. For this purpose expert were asked to rate each item from 0-5 likert scales where 0 mean not at all and 5 mean very much. There were 10 experts and scoring ranges from 0-50. A League table was made by arranging the items ascending to descending according to the rating given by expert. All those items were retained which got $50 \%$ of the agreement from the experts. Based on this study, a list of 30 items was related and 10 items were excluded.

Participants and procedure: The objective of this phase was to test the layout, instruction and readability of the items. Scale was converted in self-report measure e.g. (0-4) ratings. A sample of 20 (10 men and 10 women) was participated. No ambiguity and difficulty in understanding of the scale was reported.

\section{PSYCHOMETRIC PHASE}

Participants: The participants were 200 doctors (100 men and 100 women) age range from 22 to 36 ( $\mathrm{M}=$ $26.17, \mathrm{SD}=1.83$ ) from five Govt. hospitals of Lahore. Participants were recruited by using stratified sampling techniques as gender wise (100 men and 100 women) and designation wise (100 PG and 100 $\mathrm{HO}$ ). The exclusion criteria comprised of exclusion of all those doctors who were on contact basis and were working in both Govt. and private sectors.

\section{Ethical Considerations}

The institutional ethical committee reviewed the project for any ethical considerations. Permission was granted from the medical superintendent of the hospital and the head of respective departments to conduct the study. Purpose of the study was explained and confidentiality was assured to the participants.

\section{MEASURES}

Mental Toughness Scale (MTS) for doctors: The researcher designed and administered a questionnaire, which contain 30 items based on four point rating scale to rate the level of mental toughness and its effects on mental health problems on doctors. The options including ( 0 not at all, 1 for rarely, 2 for to some extent and 3 for often). All the participants had to rate each item according to their response to situations.

Depression, Anxiety, Stress Scale- Short Form (DASS): DASS was used to determine the discriminant validity of MTS. DASS was included 21 items and the participants had to rate each item on the level of severity of the problem on four point rating scale $^{20}$. Internal consistency of the DASS was found .81 .

Procedure: All the selected hospitals were personally visited and were briefed about the aim and objectives of the research, confidentiality about data collection was ensured to them. The participants were selected and they were told about the aim of the research, confidentiality was ensured to them that their information will be used only for the purpose of research and no personal benefits will be extracted from that, after taking their consent to participate they were presented final protocol of the research and they were instructed to carefully read the statements of each form and filled them by keeping in view the presented items, the protocol was filled in individual meeting with the participants. After taking back the forms the participants were paid regards and they were appreciated for their participation. The Statistical Package of Social Sciences (SPSS 21V) was used to analyze the data. Descriptive analysis was used to describe the characteristic features of the participants. Exploratory Factor Analysis was used to determine the factor structure of MTS. Correlation analysis was run to determine the discriminant relationship between MTS and DASS.

\section{RESULTS}

Factor analysis was carried out by using varimax rotation to explore the dimensions of mental toughness in doctors. Pre-requisites of the factor analysis was followed as cronbach's alpha was .73, KMO test value was .83 and the Bartlet test of sphericity was significant $(p<.001)$. Scree plot and Eigen value greater than 1 criteria was used to determine the number of factors and three factors 
solution was found to be the best fit model for the scale as having no dubious of items and the variance between the factor was more with this model.

FIGURE I: SCREE PLOT SHOWING FACTORS OF MENTAL TOUGHNESS SCALE FOR DOCTORS

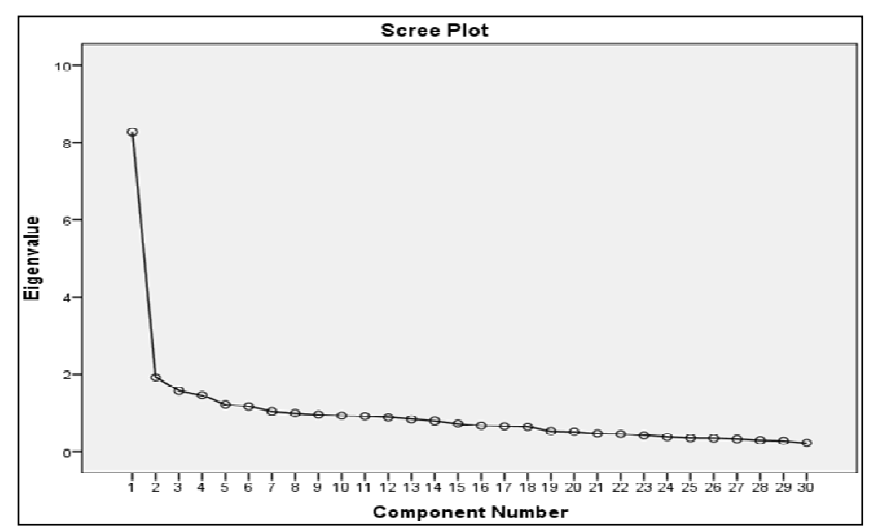

Factor 2: Resilience and Perseverance. The second factor comprised 12 items. The factors describing those items which expressing the ability to bounce back, the ability to fight back and having patience as the items included in it were "to take initiative in work", "having patience", "having stamina to face difficult situations".

Factor 3: Optimism. Third factor included 7 items. This factor included those items which are describing positive attitude, optimism, persistence and commitment. As the items included in it were "having positive thinking", "to take the criticism in positively", "not overwhelmed the things on the head", "take the things positive and shows responses in positive way".

Reliability and Validity

Split half reliability of Mental Toughness Scale was established by keeping in view the even and odd method which shows the strong split half reliability as

TABLE I: THE FACTORS STRUCTURE AND INTERNAL CONSISTENCY OF THIRTY ITEMS OF MENTAL TOUGHNESS SCALE

\begin{tabular}{|c|c|c|c|c|c|c|c|}
\hline Item No & F1 & F2 & F3 & Item No & F1 & F2 & F3 \\
\hline 8 & .56 & .36 & -.01 & 5 & .49 & .58 & .02 \\
\hline 9 & .59 & .41 & -.04 & 6 & .30 & .35 & -.18 \\
\hline 10 & .43 & .02 & .00 & 12 & .10 & .34 & .04 \\
\hline 11 & .44 & .44 & .16 & 13 & .48 & .50 & .04 \\
\hline 23 & .52 & .32 & .26 & 14 & .05 & .34 & .22 \\
\hline 24 & .61 & .24 & .23 & 15 & .12 & .41 & .27 \\
\hline 25 & .33 & .14 & .00 & 17 & .32 & .45 & .27 \\
\hline 26 & .56 & .11 & .28 & 18 & .33 & .38 & .21 \\
\hline 28 & .64 & .11 & .20 & 16 & .13 & .19 & .30 \\
\hline 29 & .68 & .18 & .14 & 19 & -.11 & .18 & .70 \\
\hline 30 & .64 & .11 & .28 & 20 & .06 & .01 & .74 \\
\hline 1 & .11 & .77 & .14 & 21 & .24 & .22 & .56 \\
\hline 2 & .14 & .75 & .12 & 22 & .13 & -.06 & .36 \\
\hline 3 & .15 & .76 & .08 & 27 & .10 & .06 & .53 \\
\hline 4 & .32 & .63 & .06 & 7 & .14 & .22 & .58 \\
\hline Eigen Value & 15.35 & 30.56 & 39.18 & Internal Consistency & .73 & .78 & .71 \\
\hline
\end{tabular}

Factor I: Determination. In the first factor 11 items were loaded. Its included those items which are describing determination, motivation and the abilities to do something as the items were, "to concentrate on the work", "having ability to learn more" and "strive to fulfill their goals and to achieve those goals". $r=0.74, p<0.001$. Test retest reliability was established with One week's interval on $20 \%(n=40)$ sample and that was $r=0.75, p<0.001$.

Content validity: It was established through the expert validation stage in the phases of scale 
development.

Discriminant validity: Discriminant validity of mental toughness scale was established with Depression Anxiety Stress Scale (DASS). High discriminant validity was found between both scales.

\section{Gender and Mental Toughness}

Independent sample t- test was used to see the mean differencein gender. Result indicated that there is no significant gender difference was found on three factors of mental toughness as determination, resilience and optimism $(p>.05)$. Only difference was found on MTS-F3 (Optimism) which shows significant results that men are more optimistic as compare to women.

\section{Job Designation and Mental Toughness}

Independent sample t- test was used to see the designation difference with three factors of MTS as determination, resilience and optimism. Results indicated that only significant difference was found on MTS-F2 (Resilience) which shows that the Post Graduate doctors are more resilient than the House Officers $(p<.001)$.

\section{DISCUSSION}

The emergence of positive psychology and introduction of strength based model has been a very significant turning point in the study of developmental psychopathology ${ }^{1}$. The excessive attention to identifying mental health problems shifted to discovering those factors which leads to promote mental health and protect the individual against developing psychological dysfunction. From the last ten years positive psychology get tremendous growth $^{5}$, this focuses on the concept that people try to show the best within them and leads towards health and happier life styles and remains it continue to touch the highest level of satisfaction and success.

Medical Profession is said to be the most oldest and a stressful profession. It is always seen that doctors performs immensely stressful jobs including long duty hours, shift duties and so on that may be emotionally very draining for them and that might affect their job performance ${ }^{20}$. Therefore, it is pertinent not only to identify but also cultivate some positive traits like mental toughness that can increase their job performance and buffer against mental health problems ${ }^{7}$.

The current study is one of the first attempt in Pakistan to develop a reliable and valid scale to measure mental toughness in doctors. Started with an emic approach phenomenology of mental toughness was explored and converted into likert type self report measure (Mental Toughness Scale (MTS) for doctors). The factor structure revealed three components of mental toughness in Pakistani culture namely Determination, resilience and optimism.
In Determination those items which are describing determination, motivation and the abilities to do something as the items were included as "to concentrate on the work", "having ability to learn more", "strive to fulfill their goals and to achieve those goals". In the second factor resilience and perseverance of those items were included which expressing the ability to bounce back, the ability to fight back and having patience as the items included in it were "to take initiative in work", "having patience", "having stamina to face difficult situations". In the optimism third factor, those items which are describing positive attitude, optimism, persistence and commitment were included. As the items in it were "having positive thinking", "to take the criticism positively", "not overwhelmed the things", "take the things positively and shows responses in positive way". Psychometrics of the scale were established which shows that Mental Toughness Scale has a satisfactory reliability and validity.

\section{CONCLUSION}

The current study was first attempt to develop a scale for the understanding and measurement of mental toughness in doctors. This research highlighted the three factors of mental toughness as determination, resilience and optimism which is the depiction of mental toughness among doctors. These factors can be further use as a base for counseling and preventing medical professional from burn out and serious mental health problems. Further study can be conduct with the doctors of govt. and private institutes to see the comparison between both groups. Studies should be conduct further to see the level of experience and expression of mental toughness among married and un-married doctors because culturally the environment of family impacts the person on each step.

Ethical permission: University of Management and Technology (Institute of Clinical Psychology) letter No. ICPRRC-201707. Dated: 03-03-2019.

Conflict of Interest: There was no conflict of interest involved for the current research.

Funding: There was no funding involved for the current research.

\section{REFERENCES}

1. Papageorgiou KA, Malanchini $M$, Denovan $A$, Clough PJ, Shakeshaft N, Schofield K, et al. Longitudinal associations between narcissism, mental toughness and school achievement. Pers Individ Dif. 2018; 131: 105-110.doi: 10.1016/ j.paid.2018.04.024.

2. Powell AJ, Myers TD. Developing mental toughness, Lesson from paralympians. Front Psychol. 2017; 8: 1270. doi: 10.3389/fpsyg.2017.01270. 
3. Erciş S. Effects of physical fitness and mental hardness on the performance of elite male basketball players. J Educ Train Stud. 2018; 6 (9a): 56-60. doi:10.11114/jets.v6i9a.3526

4. Golby J , Sheard M. Mental toughness and hardiness at different levels of rugby league. Pers Individ Diff. 2004; 37(1): 933-42.

5. Williams N, Horrell L, Edmiston D, Brady M. The impact of positive psychology on higher education. The William \& Mary Education Review. 2018; 5(1): 83-94.

6. Kruger E. Mental toughness is a predictor of suicidality in university students. Education and Health. 2018; 36(2):42-47.

7. Meggs J, Chen M. Mental toughness and attributions of failure in high performing male and female swimmers. J Human Sport Exercise. 2018; 13(2): $276-284$.

8. Sivrikaya $\mathrm{MH}$. The role of psychological hardiness on performance of scissors kick. J Educ Train Stud. 2019; 6(12a): 70-74. doi:10.11114/ jets.v6i12a.3932.

9. Gucciardi DF, Gordon S, Dimmock JA, Mallett CJ. Understanding the coach's role in the development of mental toughness: Perspectives of elite Australian football coaches. J Sports Sci. 2009; 27(13): 1483-96. doi:10.1080/ 02640410903150475.

10. St-Claire Thomson $H$, Bugler $M$, Robinson J, Clough P, McGeown SP, Perry J. Mental toughness in education: exploring relationships with attainment, attendance, behaviour and peer relationships. Educ Psychol. 2015; 35(7): 886907. doi:10.1080/01443410.2014.895294.

11. Ekmekçi R, Miçooğulları BO. Examination and comparison of psychological characteristics of American football players and handball players. Universal J Educ Res. 2018; 6(11): 2420-25. doi: 10.13189/ujer.2018.061104.

12. Åkerstedt T, Wright KP Jr. Sleep loss and fatigue in shift work and shift work disorder. Sleep Med
Clin. 2009; 4(2): 257-71.

13. Stock R, Lynam S, Cachia M. Academic success: the role of mental toughness in predicting and creating success. J High Educ Pedagog. 2018; 3(1): 429-33. doi: 10.1080/23752696.2018. 1507623

14. Jones MI, Parker JK. Mindfulness mediates the relationship between mental toughness and pain catastrophizing in cyclists. Eur J Sport Sci. 2018; 18(6): 872-881. doi: 10.1080/17461391. 2018.1478450 .

15. National Collaborating Centre for Mental Health (UK). Post-Traumatic stress disorder: The management of PTSD in adults and children in primary and secondary care. NICE Clinical Guidelines, No. 26. Leicester (UK): Gaskell; 2005. Available from: https://www.ncbi.nlm. nih.gov/ books/NBK56494/.

16. Jonason PK, Webster GD. The dirty dozen: A concise measure of the dark triad. Psychol Assess. 2010; 22(2): 420-32. doi: 10.1037/ a0019265.

17. Gucciardi DF, Gordon S, Dimmock JA. Advancing mental toughness research and theory using personal construct psychology. Int Rev Sport Exerc Psychol. 2009; 2(1): 54-72. doi: 10.1080/ 17509840802705938.

18. Brand S, Kalak N, Gerber M, Clough PJ, Lemola $S$, Puhse $U$, et al. During early and mid-adolescence, greater mental toughness is related to increased sleep quality and quality of life. J Health Psychol. 2014; 21(6): 905-15. doi: 10.1177/1359105314542816.

19. Subhan S, SaleemS, Mahmood Z. Mental toughness in Pakistani Cricketers: A factor analytical approach. FWU J Soc Sci. 2019; 13(1): 67-78.

20. Lovibond PF, Lovibond SH. The structure of negative emotional states: comparison of the depression, anxiety stress scale (DASS) with the beck depression and anxiety inventories. Behav Res Ther.1995; 33(3):335-43.
AUTHOR AFFILIATION:

Saba Aslam

$\mathrm{PhD}$ Scholar, Institute of Clinical Psychology

University of Management and Technology

Lahore, Punjab-Pakistan.

Dr. Sadia Saleem (Corresponding Author) Associate Professor, Institute of Clinical Psychology University of Management and Technology UMT Road, C-II Johar Town, Lahore, Punjab-Pakistan. Email: sadia.saleem@umt.edu.pk

\section{Dr. Zahid Mahmood}

Professor, Institute of Clinical Psychology University of Management and Technology Lahore, Punjab-Pakistan. 